\title{
Natriuretic peptide-directed medical therapy: a systematic review
}

\author{
Christella S. Alphonsus $^{1 *}$ (D, Pooveshnie Govender ${ }^{2}$, Reitze N. Rodseth ${ }^{3}$ and Bruce M. Biccard ${ }^{1}$
}

\begin{abstract}
Natriuretic peptides (NP) are strongly associated with perioperative cardiovascular events. However, in patients with raised NP, it remains unknown whether treatment to reduce NP levels prior to surgery results in better perioperative outcomes. In this systematic review and meta-analysis, we investigate NP-directed medical therapy in non-surgical patients to provide guidance for NP-directed medical therapy in surgical patients. The protocol was registered with PROSPERO (CRD42017051468). The database search included MEDLINE (PubMed), CINAHL (EBSCO host), EMBASE (EBSCO host), ProQuest, Web of Science, and Cochrane database. The primary outcome was to determine whether NP-directed medical therapy is effective in reducing NP levels within 6 months, compared to standard of care. The secondary outcome was to determine whether reducing NP levels is associated with decreased mortality. Full texts of 18 trials were reviewed. NP-directed medical therapy showed no significant difference compared to standard care in decreasing NP levels (standardized mean difference $-0.04(-0.16,0.07)$ ), but was associated with a 6-month (relative risk (RR) 0.82 (95\% confidence interval (Cl) 0.68-0.99)) reduction in mortality.
\end{abstract}

Keywords: Cardiac morbidity, Pre-operative evaluation, Myocardial ischemia

\section{Introduction}

Every year, 230 million adults undergo non-cardiac surgery worldwide (Weiser et al. 2008). In patients who are 45 years or older, $8 \%$ will suffer Myocardial Injury after Non-cardiac Surgery (MINS) (Botto et al. 2014) and 2\% will die within 30 days (Devereaux et al. 2012). MINS is typically asymptomatic without the usual features of chest pain and electrocardiogram changes seen with myocardial infarction (Botto et al. 2014). MINS has prognostic importance up to a year after surgery (Puelacher et al. 2018).

The biomarker, B-type natriuretic peptide (BNP), has been identified as an important preoperative predictor of perioperative cardiovascular events (Rodseth et al. 2014). Despite this strong association, it remains unknown whether preoperative treatment to normalize or reduce $\mathrm{NP}$ (B-type natriuretic peptide and N-terminal pro-Btype natriuretic peptide) levels prior to surgery would result in improved perioperative outcomes. This is a novel

\footnotetext{
* Correspondence: csalphonsus@gmail.com

${ }^{1}$ Consultant Anaesthesiologist, University of Cape Town, Western Cape, South Africa

Full list of author information is available at the end of the article
}

approach that has not been tested in clinical trials involving surgical patients. Thus, a systematic review of non-surgical trials is necessary to establish whether this approach is safe and effective before it can be tested in a surgical population.

The objective of this systematic review of clinical trials was to determine whether, in adults, medical patients with cardiac failure, NP-directed medical therapy is able to decrease NP levels and whether this is associated with increased survival.

These data could then be used to inform preoperative protocols aimed at decreasing NPs prior to surgery, with the intention of improving perioperative cardiovascular outcomes.

\section{Methods \\ Protocol and registration}

The protocol was registered with PROSPERO (CRD42017051468). The Preferred Reporting Items for Systematic reviews and Meta-Analysis (PRISMA) guidelines were adhered to (Moher et al. 2009).

(c) The Author(s). 2020 Open Access This article is distributed under the terms of the Creative Commons Attribution 4.0 International License (http://creativecommons.org/licenses/by/4.0/), which permits unrestricted use, distribution, and reproduction in any medium, provided you give appropriate credit to the original author(s) and the source, provide a link to the Creative Commons license, and indicate if changes were made. The Creative Commons Public Domain Dedication waiver (http://creativecommons.org/publicdomain/zero/1.0/) applies to the data made available in this article, unless otherwise stated. 


\section{Eligibility criteria}

Clinical trials of adult medical patients who were randomized to either NP guided medical therapy or standard care were eligible. We included trials which used NPs to (i) guide medical therapy in non-surgical patients, (ii) up-titrate or modify medical therapy in the response to NP levels, or (iii) included exercise as part of cardiac rehabilitation in non-surgical patients. We required that the trials report the subsequent changes in NP levels. We excluded trials that (i) monitored natriuretic peptides for prognostic or diagnostic purposes, without a strategy to lower natriuretic peptide levels, (ii) reviews of natriuretic peptide or biomarker physiology, and (iii) trials reporting natriuretic peptides in patients with acute myocardial infarction, pulmonary hypertension, cardiac resynchronization therapy, and left ventricular devices.

\section{Information sources, search, and study selection}

Three searches were conducted using search terms "brain natriuretic peptide" AND "treatment," "brain natriuretic peptide" AND "heart failure" and "brain natriuretic peptide" AND "exercise." The following databases were accessed; MEDLINE (PubMed), CINAHL (EBSCO host), EMBASE (EBSCO host), ProQuest, Web of Science, and Cochrane database. No date filter was used. An example of the search is shown in Additional file 1. The initial search was conducted on 22 December 2016 and updated on the 4 March 2018.

\section{Data collection process}

Titles were screened for potential inclusion by $\mathrm{CA}$ and PG. The abstracts of the potential papers identified through the title search were then screened using inclusion and exclusion criteria by CA and PG. The full texts of potential trials were then extracted for detailed review and analysis. Reference lists were searched for additional papers that could be included in this review. Data extraction was done by one author (CA) and then checked by a co-author. When the required data was not presented in the publication, the authors were contacted for these data.

\section{Data items}

We extracted data on the NP reduction within the first six months of randomization and mortality at 6 months. The data items extracted for this review are shown in Additional file 1: Table S1.

\section{Outcomes}

The primary outcome for this review was to determine whether a NP-directed medical therapy protocol is effective in reducing NP levels at 6 months after initiation of therapy compared to standard care. The secondary outcome was to determine whether NP-directed medical therapy decreases mortality at 6 months and at the end of the trial. The safety outcomes of changes in medical therapy were evaluated. Specific medical treatment strategies are described.

\section{Risk of bias in individual studies}

Assessment of bias in the studies was conducted by CA and verified by $\mathrm{BB}$ following discussion. Each randomized trial was assessed using the Cochrane Collaboration risk of bias tool, assessing selection bias, concealment bias, performance bias, detection bias, attrition bias, and other bias. Studies were assessed as having a low, unclear, or high risk of bias.

\section{Summary measures and synthesis of results}

The statistical analyses were conducted using Review Manager Version 5.3 (Copenhagen: The Nordic Cochrane Centre, The Cochrane Collaboration, 2014). Those trials which had data on NP levels within 6 months of therapy initiation were analyzed using standardized mean difference (SMD), and these data are presented as a forest plot. Mean and standard deviation (SD) of NP levels were used and those trials which reported NP levels as the median and interquartile range (IQR) were converted to mean and SD, using the formula proposed by Wan et al. (Wan et al. 2014). Reporting the SMD allowed for the inclusion of all trials, whether BNP or NT-proBNP, was used to monitor the medical therapeutic response. SMD addresses the difference in the effect size for an intervention when the units of measurement differ between trials e.g. use of BNP or NT-proBNP. The SMD is the difference between groups in the mean endpoint divided by the SD of the control group or pooled SD of the treatment and control groups (Hedges' $g$ ) (Guyatt et al. 2015).

A meta-analysis of mortality within 6 months of the initiation of therapy, with subgroup analyses at 4 and 6 months was conducted. The results are reported as relative risk (RR), with 95\% confidence intervals (CI), and presented as forest plots. Random effects models were used where the $I^{2}$ statistic $>2$ s5\% (representing significant heterogeneity), otherwise a fixed-effects model was used.

\section{Risk of bias across studies}

Risk of bias across studies was assessed with funnel plots for NP reduction and mortality.

\section{Post hoc}

After extracting and analyzing the data, it was noticed that the methodology used in the exercise trials differed significantly from the medical therapy trials. This difference was so substantial that we deemed it inappropriate 
to pool the two interventions. We therefore made a post hoc decision to separate the exercise studies from the medical therapy studies. These exercise study data are presented in the accompanying publication (CS Alphonsus et al. 2019).

\section{Results}

\section{Study selection}

Sixty-four full-text articles were reviewed for potential inclusion and 26 trials (presented in 27 publications) met the inclusion criteria. An additional eight trials were added from references (Fig. 1). Eighteen trials of medical therapy interventions were identified (reported in 19 publications; 1 trial was reported in 2 separate papers) (Maeder et al. 2013; Pfisterer et al. 2009) fulfilled the inclusion criteria, although only 14 trials provided data for this review's outcomes. The 16 exercise trials were subsequently removed from this review, following the post hoc decision to present these trials in a separate paper (CS Alphonsus et. al. 2019).

We evaluated previous systematic reviews identified in the search using the AMSTAR format (Additional file 1: Table S2).

\section{Study characteristics of included studies}

The characteristics of the included clinical trials are shown in Table 1. These trials included adult patients of 18 years and older. The majority of the trials examined outpatient NP-directed medical therapy, with follow-up of 15 months or more. In 10 out of 18 trials, patients were seen by a specialist at a clinic (Anguita et al. 2010; Berger et al. 2010; Eurlings et al. 2010; Januzzi et al. 2011; Jourdain et al. 2007; Lainchbury et al. 2009; Murdoch et al. 1999; Persson et al. 2010; Schou et al. 2013; Shah et al. 2011; Troughton et al. 2000). Most patients were randomized once heart

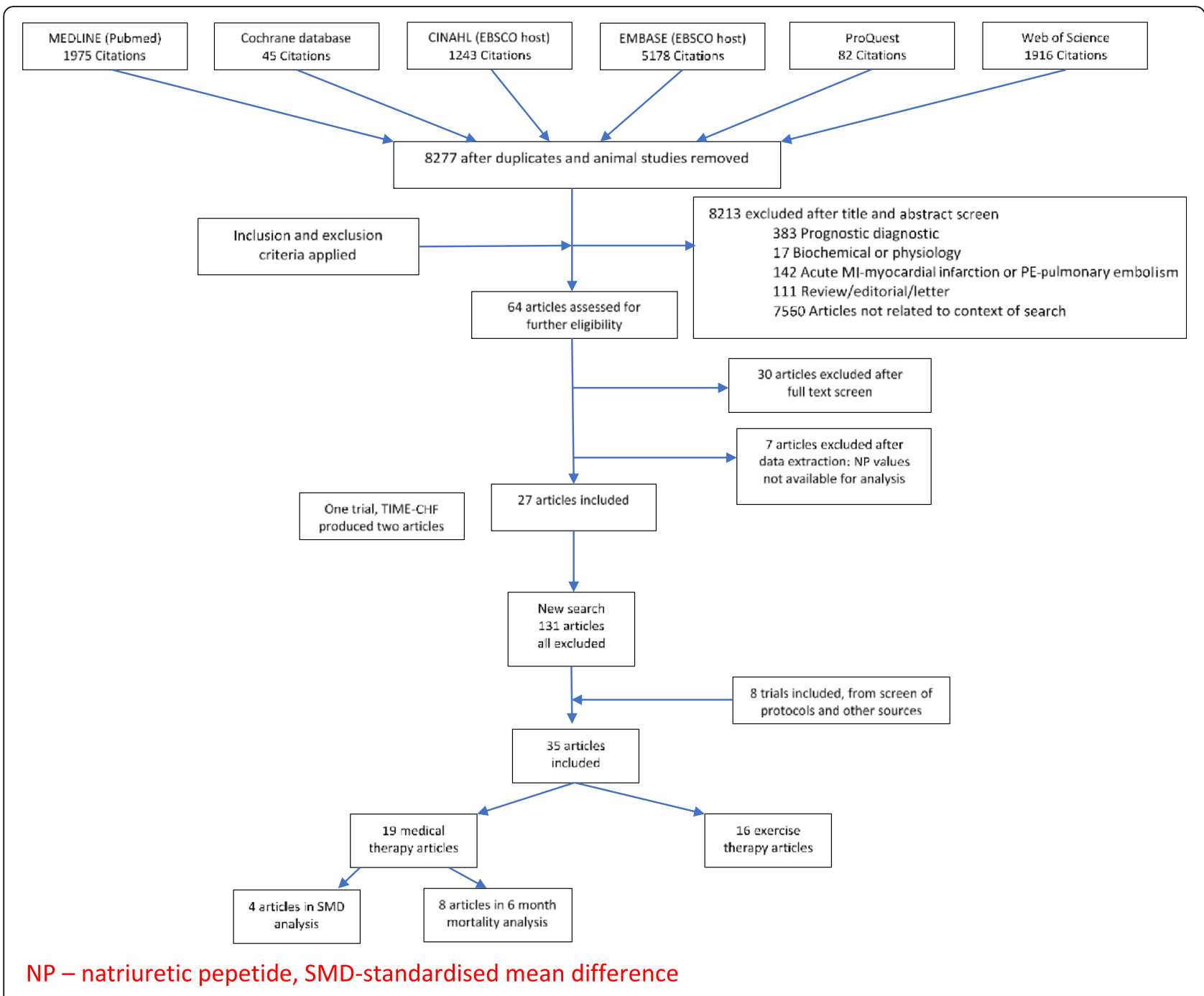

Fig. 1 PRISMA flow diagram 
Table 1 Characteristics of included clinical trials

\begin{tabular}{|c|c|c|c|}
\hline Clinical trial & Patients & $\begin{array}{l}\text { Intervention arm }(n) \text { vs } \\
\text { standard care arm }(n) \dagger\end{array}$ & Follow-up (months) \\
\hline Murdoch et al. (1999) & Stable CHF, LVEF $\leq 35 \%$ & $\begin{array}{l}\text { BNP arm } n=10 \\
\text { Standard care } n=10\end{array}$ & 2 \\
\hline Troughton et al. (2000) & Decompensated HF now stabilised, LVEF $<40 \%$ & $\begin{array}{l}\text { BNP arm } n=33 \\
\text { Standard care } n=36\end{array}$ & 9.5 \\
\hline Beck-da-Silva et al. (2005) & $>18$ years, stable CHF but not on $\beta$ blockers, LVEF $\leq 40 \%$ & $\begin{array}{l}\text { BNP arm } n=21 \\
\text { Standard care } n=20\end{array}$ & 3 \\
\hline Jourdain et al. (2007) & $>18$ years, optimized on treatment, LVEF < 45\% & $\begin{array}{l}\text { BNP arm } n=110 \\
\text { Standard care } n=110\end{array}$ & 15 \\
\hline Ozkara et al. (2007) & Treated with ACEI/loop diuretic, LVEF $\leq 50 \%$ & $\begin{array}{l}\text { NT-proBNP arm } n=79 \neq \\
\text { Standard care } n=61\end{array}$ & 6 \\
\hline Pfisterer et al. (2009) & $\begin{array}{l}\geq 60 \text { years, LVEF } \leq \mathrm{v} 45 \%, 60-74 \text { years=NT-proBNP } \\
\geq 400 \mathrm{pg} / \mathrm{ml} ; \geq 75 \text { years }=\text { NT-proBNP } 800 \mathrm{pg} / \mathrm{ml}\end{array}$ & $\begin{array}{l}\text { NT-proBNP arm } n=251 \\
\text { Standard care } n=248\end{array}$ & 18 \\
\hline Lainchbury et al. (2009)* & $>18$ years, AHF now stabilised & $\begin{array}{l}\text { NT-proBNP arm } n=121 \\
\text { Standard care } n=122\end{array}$ & 36 \\
\hline Anguita et al. (2010) & $>18$ years, AHF & $\begin{array}{l}\text { BNP arm } n=30 \\
\text { Standard care } n=30\end{array}$ & 18 \\
\hline Persson et al. (2010) & $\begin{array}{l}\text { LVEF }<50 \% \text {, males NT-proBNP }>800 \mathrm{ng} / \mathrm{ml} \text {, females }> \\
1000 \mathrm{ng} / \mathrm{ml}\end{array}$ & $\begin{array}{l}\text { NT-proBNP arm } n=125 \\
\text { Standard care } n=127\end{array}$ & 9 \\
\hline Eurlings et al. (2010) & $\begin{array}{l}\text { AHF NT-proBNP }>1700 \text {, randomized at discharge if }> \\
10 \% \text { drop in NT-proBNP }\end{array}$ & $\begin{array}{l}\text { NT-proBNP arm } n=174 \\
\text { Standard care } n=171\end{array}$ & 24 \\
\hline Berger et al. (2010)* & AHF now stabilised, LVEF < 40\% & $\begin{array}{l}\text { NT-proBNP }+ \text { MC arm (only } \\
\text { patients with NT-proBNP }>2200 \\
\text { pg/ml) } n=92 \\
\text { Standard care } n=90\end{array}$ & Maximum 18; minimum 12 \\
\hline Januzzi Jr et al. (2011) & $>21$ years, LVEF $<40 \%$ & $\begin{array}{l}\text { NT-proBNP arm } n=75 \\
\text { Standard care } n=76\end{array}$ & 10 \\
\hline Shah et al. (2011) & Decompensation HF now stabilized, LVEF $\leq 35 \%$ & $\begin{array}{l}\text { BNP arm } n=68 \\
\text { Standard care } n=69\end{array}$ & 4 \\
\hline Karlstrom (2011) & $\begin{array}{l}>18 \text { years; BNP }>150 \mathrm{ng} / \mathrm{L} \text { for those aged }<75 \text { years, } \\
\text { and } \mathrm{BNP}>300 \mathrm{ng} / \mathrm{L} \text { for those aged }>75 \text { years }\end{array}$ & $\begin{array}{l}\text { BNP arm } n=147 \\
\text { Standard care } n=132\end{array}$ & 33 \\
\hline Maeder et al. (2013) & $\begin{array}{l}\geq 60 \text { years, LVEF }>45 \%, 60-74 \text { years }=\text { NT-proBNP } \geq \\
400 \mathrm{pg} / \mathrm{ml} ; \geq 75 \text { years }=\text { NT-proBNP } 800 \mathrm{pg} / \mathrm{ml}\end{array}$ & $\begin{array}{l}\text { NT-proBNP arm } n=59 \\
\text { Standard care } n=64\end{array}$ & 18 \\
\hline Schou et al. (2013) & $\begin{array}{l}\text { > 18years, Optimised on treatment and implantable } \\
\text { ICD/CRT, LVEF }<45 \% \text {, NT-proBNP }>1000\end{array}$ & $\begin{array}{l}\text { NT-proBNP arm } n=199 \\
\text { Standard care } n=208\end{array}$ & Median 30 \\
\hline Carubelli et al. (2016) & Randomized after stabilization of AHF & $\begin{array}{l}\text { NT-proBNP arm } n=137 \\
\text { Standard care } n=134\end{array}$ & Mean 18 \\
\hline Stienen et al. (2018) & $\begin{array}{l}\text { Decompensated HF, NT-proBNP levels > } 1700 \mathrm{ng} / \mathrm{ml} \\
\text { within } 24 \mathrm{~h} \text { of hospital admission. In hospital intervention }\end{array}$ & $\begin{array}{l}\text { NT-proBNP arm } n=201 \\
\text { Standard care } n=203\end{array}$ & 6 \\
\hline Felker et al. (2017) & LVEF $\leq 40 \%$, NT-proBNP > 2000 pg/mL/BNP > 400 pg/ml & $\begin{array}{l}\text { NT-proBNP arm } n=446 \\
\text { Standard care } n=448\end{array}$ & 12 \\
\hline
\end{tabular}

CHF chronic heart failure, $A H F$-acute heart failure, NT-proBNP N-terminal pro b-type natriuretic peptide, $L V E F$ left ventricular ejection fraction, $A R B$ angiotensin II receptor blocker, $A C E I$ angiotensin converting enzyme inhibitor, $A R A$ aldosterone receptor antagonist, $B$-blocker beta blocker, ICD/CRT implantable converter defibrillator/cardiac resynchronisation therapy, BNP B-type natriuretic peptide, MC multidisciplinary care, NYHA New York Heart Association, HF heart failure tCheck Additional file 1

*Randomised to three-arm but only 2 meet the inclusion criteria for this review, NP-directed arm and control arm most reflecting usual patient care ₹ Only patients in the intervention arm received spironolactone

failure was medically stabilized and 12 out of 18 trials enrolled patients with EF $\leq 50 \%$ (Beck-da-Silva et al. 2005; Felker et al. 2017; Januzzi et al. 2011; Jourdain et al. 2007; Karlstrom et al. 2011; Murdoch et al. 1999; Ozkara et al. 2007; Persson et al. 2010; Schou et al. 2013; Shah et al. 2011; Stienen et al. 2018; Troughton et al. 2000). The remainder of the trials combined patients with preserved and reduced ejection fraction
(Anguita et al. 2010; Berger et al. 2010; Carubelli et al. 2016; Eurlings et al. 2010; Lainchbury et al. 2009; Maeder et al. 2013; Pfisterer et al. 2009).

The conduct of the trial intervention arms is shown in Table 2. All trials randomized patients into NP-directed medical therapy or clinical/usual care. Two trials were three-arm trials, but for this analysis, only the interventional and usual care arms were included (Berger et al. 
Table 2 The conduct of the natriuretic-peptide (NP)-directed clinical trials

\begin{tabular}{|c|c|c|c|}
\hline $\begin{array}{l}\text { Clinical } \\
\text { Trial }\end{array}$ & Level of care in interventional group & Frequency of visits & NP target \\
\hline Murdoch & Specialist HF clinic & Every 2 weeks & Single target $\mathrm{BNP}<50 \mathrm{pg} / \mathrm{ml}$ \\
\hline Troughton & Specialist HF clinic & Every 3 months & Single target N-BNP $<200 \mathrm{pmol} / \mathrm{L}$ \\
\hline $\begin{array}{l}\text { Beck-da- } \\
\text { Silva }\end{array}$ & Nurse-led HF clinic & Every 3 months & $\begin{array}{l}\text { Individualized according to } \\
\text { symptoms in relation to BNP levels. }\end{array}$ \\
\hline Jourdain & Specialist care at the clinic & 1 month (for 3 months) then 3 months & Single target $B N P<100 \mathrm{pg} / \mathrm{ml}$ \\
\hline Ozkara & Physician clinic visits & Treatment not adjusted throughout study & No BNP target set \\
\hline $\begin{array}{l}\text { Lainchbury } \\
*\end{array}$ & $\begin{array}{l}\text { Research clinic (with possible specialist } \\
\text { input) }\end{array}$ & Every 3 months & $\begin{array}{l}\text { Single target NT-proBNP }<150 \\
\mathrm{pmol} / \mathrm{L}\end{array}$ \\
\hline $\begin{array}{l}\text { Maeder; } \\
\text { Pfisterer }\end{array}$ & Outpatients visits & $1,3,6,12,18$ months & $\begin{array}{l}\text { NT-proBNP }<400 \mathrm{pg} / \mathrm{ml} \text { in }<75 \text { years } \\
\text { and }<800 \mathrm{pg} / \mathrm{ml} \text { in } \geq 75 \text { years }\end{array}$ \\
\hline Eurlings & Specialist care at the clinic & 2 weeks, 1 month, then 3 months & $\begin{array}{l}\text { Individualized NT-proBNP }<10 \% \text { of } \\
\text { randomization level }\end{array}$ \\
\hline Berger * & HF specialist clinic & $\begin{array}{l}\text { Every } 2 \text { weeks till NT-proBNP target met. Then as } \\
\text { required. }\end{array}$ & $\begin{array}{l}\text { Single target NT-proBNP } \\
<2200 \mathrm{pg} / \mathrm{ml}\end{array}$ \\
\hline Persson & Primary care centres & 10 days, $1,3,6,9$ months & $\begin{array}{l}\text { Individualized NT-proBNP }<50 \% \\
\text { from baseline level }\end{array}$ \\
\hline Anguita & Cardiology clinic & $1,2,3,6,24,18$ months & Single target $\mathrm{BNP}<100 \mathrm{pg} / \mathrm{ml}$ \\
\hline Shah & HF clinic with specialist input & 1 week, 1, 2, 3, 4 months after discharge & $\begin{array}{l}\text { Individualized BNP }<2 \text { times } \\
\text { discharge level }\end{array}$ \\
\hline Januzzi & HF clinic & Every 3 months & $\begin{array}{l}\text { Single target NT-proBNP } \leq \\
1000 \mathrm{pg} / \mathrm{ml}\end{array}$ \\
\hline Karlstrom & Outpatient visits & $2,6,10,16,2,36,48$ weeks, then every 6 months & $\begin{array}{l}<75 \text { years }(\mathrm{BNP}<15 \mathrm{ng} / \mathrm{L}) \\
\text { and } \geq 75 \mathrm{yrs}(\mathrm{BNP}<300 \mathrm{ng} / \mathrm{L})\end{array}$ \\
\hline Schou & Specialist heart failure clinic & Every $1-3$ months & $\begin{array}{l}\text { Individualised NT-proBNP }<30 \% \\
\text { of randomization level }\end{array}$ \\
\hline Carubelli & $\begin{array}{l}\text { Single center, initially in hospital } \\
\text { management and then outpatient } \\
\text { visits }\end{array}$ & $\begin{array}{l}\text { Frequent visits if NT-proBNP still raised after discharge. } \\
\text { Then telephonic follow up at } 1,3 \text {, and } 6 \text { months }\end{array}$ & $\begin{array}{l}\text { Single target NT-proBNP } \leq 3000 \\
\mathrm{pg} / \mathrm{ml}\end{array}$ \\
\hline Felker & Outpatient visits & 2 and 6 weeks, then every 3 months & $\begin{array}{l}\text { Single target NT-proBNP } \\
<1000 \mathrm{pg} / \mathrm{mL} \text {. }\end{array}$ \\
\hline Stienen & Intervention carried out in the hospital & 1 week and at 1, 3, and 6 months & $\begin{array}{l}\text { Individualized to reduce } \\
\text { NT-proBNP by at least 30\% by } \\
\text { discharge }\end{array}$ \\
\hline
\end{tabular}

NP natriuretic peptide, NT-proBNP N-terminal pro B-type natriuretic peptide, LVEF left ventricular ejection fraction, $A R B$ angiotensin II receptor blocker, ACElangiotensin-converting enzyme inhibitor, BNP B-type natriuretic peptide, NYHA New York Heart Association, $H F$ heart failure

*Lainchbury and Berger: three-arm trial but only NT-proBNP guided management group and usual care group compared

2010; Lainchbury et al. 2009). In the majority of trials, the NP threshold for inclusion was consistent across age and gender, with the exception of three trials, where the threshold was either age- or gender-specific (Karlstrom et al. 2011; Maeder et al. 2013; Persson et al. 2010; Pfisterer et al. 2009). Nine trials set population NP targets (Anguita et al. 2010; Berger et al. 2010; Carubelli et al. 2016; Felker et al. 2017; Januzzi et al. 2011; Jourdain et al. 2007; Lainchbury et al. 2009; Murdoch et al. 1999; Troughton et al. 2000), eight trials set individualized NP targets (Beck-da-Silva et al. 2005; Eurlings et al. 2010; Karlstrom et al. 2011; Maeder et al. 2013; Persson et al. 2010; Pfisterer et al. 2009; Schou et al. 2013; Shah et al. 2011; Stienen et al. 2018), and one trial had no set NP target, but directed medical therapy to reduce the NP level (Ozkara et al. 2007). The management of the standard care arms is shown in Additional file 1.

Two trials were stopped early (Felker et al. 2017; Januzzi et al. 2011), Felker et. al for the benefit, and Januzzi et al. for futility.

\section{Risk of bias within studies and across studies}

The risk of bias of the included trials is shown in Additional file 1: Figures S1 and S2. The random sequence generation was unclear in half the trials, and blinding of patients and investigators was low. Many trials did not clearly document if outcome assessors were blinded. The funnel plots for SMD (Additional file 1: Figure S3), and 6-month mortality (Additional file 1: Figure S4) did not suggest publication bias. 
Results of individual studies and synthesis of results The efficacy of an NP-directed medical therapy in reducing NP levels within 6 months compared to standard care Fourteen out of 18 medical therapy trials presented data on change in NP levels during the trial (Anguita et al. 2010; Carubelli et al. 2016; Eurlings et al. 2010; Felker et al. 2017; Januzzi et al. 2011; Jourdain et al. 2007; Karlstrom et al. 2011; Lainchbury et al. 2009; Maeder et al. 2013; Murdoch et al. 1999; Persson et al. 2010; Pfisterer et al. 2009; Schou et al. 2013; Shah et al. 2011; Stienen et al. 2018; Troughton et al. 2000), of which 7 out of 14 trials presented data on NP levels within the first 6 months of the trial (Anguita et al. 2010; Carubelli et al. 2016; Felker et al. 2017; Maeder et al. 2013; Pfisterer et al. 2009; Shah et al. 2011; Stienen et al. 2018). Three trials Shah, Carubelli, and Stienen were excluded as the data was reported at differing time points before 6 months: Stienen (mean $12 \pm 10$ days) (Stienen et al. 2018), Carubelli (mean $11 \pm 9$ days) (Carubelli et al. 2016) and Shah (4 months) (Shah et al. 2011). The overall point estimate of the four remaining trials was nonsignificant at 6 months of NP-directed medical therapy with low heterogeneity in the included trials (Fig. 2), (SMD - 0.04, 95\% CI - 0.16, 0.07).

\section{Reduction in NP levels and its association with mortality}

Seventeen out of 18 studies reported mortality at trial completion (Anguita et al. 2010; Beck-da-Silva et al. 2005; Berger et al. 2010; Carubelli et al. 2016; Eurlings et al. 2010; Felker et al. 2017; Januzzi et al. 2011; Jourdain et al. 2007; Karlstrom et al. 2011; Lainchbury et al. 2009; Maeder et al. 2013; Ozkara et al. 2007; Persson et al. 2010; Pfisterer et al. 2009; Schou et al. 2013; Shah et al. 2011; Stienen et al. 2018; Troughton et al. 2000). After extracting the end of trial mortality data, it was deemed inappropriate to conduct a meta-analysis, as the duration of the trial follow up periods differed between the trials. It was therefore impossible to conduct a metaanalysis at a fixed long-term time point.
Eight of the 18 trials (Anguita et al. 2010; Eurlings et al. 2010; Felker et al. 2017; Lainchbury et al. 2009; Maeder et al. 2013; Pfisterer et al. 2009; Shah et al. 2011; Stienen et al. 2018) reported mortality within the first 6 months of the intervention. Two trials reported mortality at 4 months, and 6 trials reported mortality at 6 months. NP-directed medical therapy was associated with a reduction in mortality within the first 6 months of the intervention (RR 0.82, 95\% CI 0.68-0.99). Subgroup analysis suggested little heterogeneity between the 4-month and 6-month outcomes (Fig. 3).

\section{Adverse events in relation to the change in therapy}

Seven out of 18 studies recorded adverse effects of medical therapy on electrolytes and the cardiovascular system (Eurlings et al. 2010; Januzzi et al. 2011; Jourdain et al. 2007; Maeder et al. 2013; Murdoch et al. 1999; Pfisterer et al. 2009; Shah et al. 2011; Troughton et al. 2000). These were deemed not serious and six of these studies showed no difference in the incidence of adverse effects of therapy between the intervention and control groups.

\section{The specific treatment strategies used in the trials}

The treatment strategies and efficacy of these treatments varied between the trials. The two trials that showed the most benefit associated with NP-directed medical therapy,(Carubelli et al. 2016) and (Shah et al. 2011), showed efficacy for diuretics (the former) and angiotensinconverting enzyme inhibitors and beta-blockers (the latter). The two studies that showed the greatest number of patients reaching target NP levels, (Lainchbury et al. 2009) and (Karlstrom et al. 2011), showed that a combination of therapies was effective, including diuretics, angiotensin-converting enzyme inhibitor/angiotensin II receptor blockers and beta-blockers.

\section{Discussion}

The principal findings of this systematic review are that NP-directed medical therapy does not significantly reduce NP levels at 6 months after initiation of NP-

\begin{tabular}{|c|c|c|c|c|c|c|c|c|c|c|}
\hline \multirow[b]{2}{*}{ Study or Subgroup } & \multicolumn{2}{|c|}{ Intervention } & \multicolumn{4}{|c|}{ Control } & \multicolumn{3}{|c|}{ Std. Mean Difference } & \multirow{2}{*}{$\begin{array}{l}\text { Std. Mean Difference } \\
\text { IV, Fixed, } 95 \% \mathrm{Cl}\end{array}$} \\
\hline & Mean & SD & Total & Mean & SD & Total & Weight & IV, Fixed, $95 \% \mathrm{Cl}$ & Year & \\
\hline Pfisterer 2006 & $3,345.7$ & 4,260 & 211 & $4,223.7$ & $5,573.2$ & 192 & $34.8 \%$ & $-0.18[-0.37,0.02]$ & 2006 & \\
\hline Anguita 2009 & 73.9 & 87.6 & 28 & 102 & 195.3 & 28 & $4.8 \%$ & $-0.18[-0.71,0.34]$ & 2010 & \\
\hline Maeder 2013 & $3,676.7$ & $6,344.5$ & 38 & $3,474.2$ & $4,445.6$ & 45 & $7.2 \%$ & $0.04[-0.39,0.47]$ & 2013 & \\
\hline Felker 2017 & $2,047.7$ & 2,645 & 305 & $1,925.7$ & 2,093 & 307 & $53.2 \%$ & $0.05[-0.11,0.21]$ & 2017 & \\
\hline Total $(95 \% \mathrm{Cl})$ & & & 582 & & & 572 & $100.0 \%$ & $-0.04[-0.16,0.07]$ & & \\
\hline \multicolumn{10}{|c|}{$\begin{array}{l}\text { Heterogeneity: } C h{ }^{2}=3.58, d f=3(P=0.31) ; l^{2}=16 \% \\
\text { Test for owerall effect: } Z=0.69(P=0.49)\end{array}$} & $\begin{array}{cccc}-0.5 & -0.25 & 0.25 & 0.5 \\
\text { Intervention group Control group }\end{array}$ \\
\hline \multicolumn{11}{|c|}{ SD-standard deviation, $\mathrm{Cl}$-confidence interval } \\
\hline \multicolumn{11}{|c|}{$\begin{array}{l}\text { Fig. 2. Efficacy of natriuretic peptide-directed medical therapy versus control in reducing BNP-levels within } 6 \text { months(Standardised mean } \\
\text { difference in natriuretic peptide levels in NP-directed medical therapy clinical trials). SD, standard deviation; Cl, confidence interval }\end{array}$} \\
\hline
\end{tabular}




\begin{tabular}{|c|c|c|c|c|c|c|c|c|c|c|c|}
\hline \multirow{2}{*}{$\frac{\text { Study or Subgroup }}{2.1 .14 \text { months }}$} & \multirow[t]{2}{*}{ Events } & Intervention & \multirow[t]{2}{*}{ Events } & \multirow[t]{2}{*}{ Total } & \multicolumn{3}{|c|}{ Risk Ratio } & \multicolumn{4}{|c|}{$\begin{array}{c}\text { Risk Ratio } \\
\text { M-H, Fixed, } 95 \% \mathrm{Cl} \\
\end{array}$} \\
\hline & & Total & & & Weight & M-H, Fixed, $95 \% \mathrm{Cl}$ & Year & & & & \\
\hline Eurlings 2010 & 15 & 174 & 18 & 171 & $8.8 \%$ & $0.82[0.43,1.57]$ & 2010 & & $\rightarrow$ & 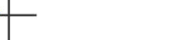 & \\
\hline $\begin{array}{l}\text { Shah } 2011 \\
\text { Subtotal }(95 \% \mathrm{Cl})\end{array}$ & 1 & $\begin{array}{r}68 \\
242\end{array}$ & 3 & $\begin{array}{r}69 \\
240\end{array}$ & $\begin{array}{r}1.4 \% \\
10.2 \%\end{array}$ & $\begin{array}{l}0.34[0.04,3.17] \\
0.75[0.40,1.40]\end{array}$ & 2011 & & & & \\
\hline $\begin{array}{l}\text { Total events } \\
\text { Heterogeneity: chiz= } \\
\text { Test for overall effect }\end{array}$ & $\begin{array}{r}16 \\
0.56, d f= \\
Z=0.90\end{array}$ & $\begin{array}{l}1(P=0 \\
P=0.37\end{array}$ & D. $46 ; i^{21}=$ & $0 \%$ & & & & & & & \\
\hline 2.1.2 6 months & & & & & & & & & & & \\
\hline Pfisterer 2006 & 41 & 251 & 51 & 248 & $24.7 \%$ & $0.79[0.55,1.15]$ & 2006 & & $\rightarrow+$ & & \\
\hline Anguita 2009 & 2 & 30 & 2 & 30 & $1.0 \%$ & $1.00[0.15,6.64]$ & 2009 & & & & \\
\hline Lainchbury 2010 & 8 & 121 & 12 & 122 & $5.8 \%$ & $0.67[0.28,1.59]$ & 2010 & & 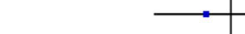 & 一 & \\
\hline Waeder 2013 & 21 & 59 & 19 & 64 & $8.8 \%$ & $1.20[0.72,2.00]$ & 2013 & & $=$ & $=-$ & \\
\hline Steinen 2017 & 7 & 201 & 13 & 203 & $6.2 \%$ & $0.54[0.22,1.33]$ & 2017 & & & + & \\
\hline $\begin{array}{l}\text { Felker } 2017 \\
\text { Subtotal }(95 \% \mathrm{Cl})\end{array}$ & 75 & $\begin{array}{r}446 \\
1108\end{array}$ & 90 & $\begin{array}{r}448 \\
1115\end{array}$ & $\begin{array}{l}43.3 \% \\
89.8 \%\end{array}$ & $\begin{array}{l}0.84[0.63,1.10] \\
0.83[0.69,1.01]\end{array}$ & 2017 & & $\rightarrow$ & & \\
\hline $\begin{array}{l}\text { Total events } \\
\text { Heterogeneity: Chiz } \\
\text { Test for overall effect }\end{array}$ & $\begin{array}{l}154 \\
3.17, \mathrm{df}= \\
Z=1.88\end{array}$ & $\begin{array}{l}5(P=0 \\
P=0.06\end{array}$ & $\begin{array}{l}187 \\
0.67) ;\left.\right|^{2}=1 \\
6)\end{array}$ & $0 \%$ & & & & & & & \\
\hline Total $(95 \% \mathrm{Cl})$ & & 1350 & & 1355 & $100.0 \%$ & $0.82[0.68,0.99]$ & & & $\bullet$ & & \\
\hline Total events & 170 & & 208 & & & & & & & & \\
\hline $\begin{array}{l}\text { Heterogeneity: Chiz } \\
\text { Test for overall effect } \\
\text { Test for subqroup di }\end{array}$ & $\begin{array}{l}3.82, \mathrm{df}= \\
Z=2.07 \\
\text { ferences: }\end{array}$ & $\begin{array}{l}7(P=0 \\
P=0.04 \\
C h)^{2}=0\end{array}$ & $\begin{array}{l}0.80) ; I^{2}=0 \\
\text { 4) } \\
\text { 1.09, } d f=1\end{array}$ & $\begin{array}{l}0 \% \\
1 \mathrm{~F}=0\end{array}$ & $.76) . I^{2}=0$ & & & 0.01 & $\begin{array}{c}0.1 \\
\text { Intervention group }\end{array}$ & 1 Control group & 100 \\
\hline
\end{tabular}

directed medical therapy. However, NP-directed medical therapy may be associated with decreased mortality in the short term, and there is little heterogeneity for this finding.

\section{Strengths}

The strength of this review is that we evaluated the efficacy of NP-directed medical therapy from clinical trials, on the biochemical response of patient NP levels, and the clinically relevant outcome of mortality. The methodology of this systematic review and meta-analysis is robust.

\section{Findings in relation to other studies}

There is an important fundamental difference between this meta-analysis, and the other two meta-analyses that were published after the protocol for our meta-analysis was registered (Khan et al. 2018; Pufulete et al. 2017). The primary outcome of our meta-analysis was to evaluate if it was possible to decrease NP levels with NPdirected therapy, while the primary outcome of the other two meta-analyses was to determine if NP-directed medical therapy was associated with a survival benefit. Evaluation of a potential survival benefit was a secondary outcome in our meta-analysis. Our primary interest was to determine whether perioperative physicians could possibly decrease NP levels prior to elective surgery in patients with high NP levels (and thereby potentially improve the risk profile of poor surgical candidates). Both these meta-analyses also had point estimates favoring survival benefit with NP-directed therapy in the long term. The importance of our meta-analysis is that (i) a reduction in NP levels is not necessarily essential to demonstrate a survival benefit with NP-directed medical therapy, and (ii) that this survival benefit may be seen earlier than what has been previously documented. The utility of NP-directed medical therapy in preoperative surgical patients is unknown, as there are currently no surgical trials in this field. This meta-analysis suggests that there is potential utility in this approach in surgical patients.

Preoperative risk stratification of high-risk patients is advocated by international guidelines, the most recent being the Canadian Cardiovascular Society Guidelines on Perioperative Cardiac Risk Assessment and Management for Patients Who Undergo Noncardiac Surgery (Duceppe et al. 2017). Screening for natriuretic peptides is a key component of risk stratification (Duceppe et al. 2017).

Our meta-analysis suggests that there may be a further benefit to the reduction of NP levels prior to surgery. The survival benefit seen with NP-directed medical therapy in this meta-analysis may suggest that an intensification of medical therapy is warranted in patients with marked physiological derangement reflected by a markedly 
elevated NP level. These high NP levels may reflect some reversibility in volume status and myocyte ischemia which is responsive to further medical therapy. Indeed, the trials which demonstrated the greatest number of patients reaching a target NP level included a combination of therapies which would have had both volume and ischemia efficacy (Karlstrom et al. 2011; Lainchbury et al. 2009). The importance of this systematic review is the following. Firstly, these findings suggest that there is potential to improve survival for an elective surgical population through NP-directed medical therapy. Secondly, the perioperative period is a powerful modifier of risk, and decreasing this risk, has the potential to change morbidity and mortality up to a year after surgery (Puelacher et al. 2018).

\section{Limitations}

We were unable to obtain data from all the included trials for the SMD, the patients reaching the target NP and the time to NP reduction analysis. This is because most trials did not publish these end-points, nor was this included as part of the trial protocols. It is possible that if we had a larger sample which included data from all trials, then we may have shown an association between NP-directed medical therapy and a reduction in NP levels. However, it appears from this meta-analysis, that it is the intensification of medical therapy, rather than the reduction in NP levels, which may be important for short-term survival.

The non-parametric data for the SMD analysis was transformed to mean and standard deviation to facilitate analysis and caution should be taken when interpreting these results. The range of starting NP level on randomization in the intervention groups is large and could dramatically influence responsiveness to NPdirected therapy. However, despite these differences in the pre-intervention NP levels, mortality decreased in the NP-directed therapy arm, with little heterogeneity. It could be argued, however, that this early mortality (i.e., at 6 months of therapy initiation) signal is fragile. If a random-effects meta-analysis is conducted, then one cannot demonstrate a survival benefit associated with NP-directed medical therapy (RR 0.88, 95\% 0.75-1.04, $p$ $=0.14$ ). Similarly, a sensitivity analysis which excludes all trials with a high risk of bias is not associated with a survival benefit (RR $0.84,95 \%$ CI $0.61-1.15, p=0.27$ ) in a random-effects model. The survival benefit demonstrated in this meta-analysis therefore should be considered "hypothesis-generating" at best. It was not possible to control for the effect of age or renal function on NP for this analysis.

Finally, the included trials had very different implemented protocols, and thus it is not possible to identify a preferred medical management plan based on these data.

\section{Future research}

This systematic review provides support for a clinical trial of preoperative NP-directed medical therapy in high-risk elective surgical patients.

\section{Conclusion}

NP-directed medical therapy does not necessarily decrease NP levels, but it may be associated with a survival benefit. There may be a place for preoperative NPdirected medical therapy in high-risk surgical patients.

\section{Supplementary information}

Supplementary information accompanies this paper at https://doi.org/10. 1186/s13741-019-0134-y

Additional file 1: Example of search strategy for the systematic review. Description of the standard care arm. Table S1. Data extracted for metaanalyses SMD - standardised mean difference, NP - natriuretic peptide. Table S2. AMSTAR evaluation of previous systematic reviews. Figure S1. Risk of bias summary. Figure S2. Risk of bias graph. Figure S3. Funnel plot for Standard Mean Difference forest plot. Figure S4. Funnel plot for mortality at 4 and 6 months forest plot.

\section{Abbreviations}

ACEl: Angiotensin-converting enzyme inhibitor; ARB: Angiotensin II receptor blocker; BB: Beta-blocker; BNP: B-type natriuretic peptide; Cl: Confidence interval; IQR: Interquartile range; MINS: Myocardial injury after non-cardiac surgery; NP: Natriuretic peptides; NT-proBNP: N-terminal pro B-type natriuretic peptide; RR: Relative risk; SD: Standard deviation; SMD: Standardized mean difference

Acknowledgements

None

Authors' contributions

Screening, extraction of articles and data extraction was done by CA, PG, and $B B$. The manuscript was prepared by $C A, B B$, and RR. All authors read and approved the final manuscript

\section{Funding}

CA has received research funding from the South African Society of Anaesthesiologists and the University of Cape Town.

Availability of data and materials

All articles available online and datasets are available from the corresponding author.

Ethics approval and consent to participate

Not applicable

Consent for publication

Not applicable.

Competing interests

The authors declare that they have no competing interests.

Author details

${ }^{1}$ Consultant Anaesthesiologist, University of Cape Town, Western Cape, South Africa. ${ }^{2}$ Anaesthesia Fellow, The Royal Papworth Hospital, Cambridgeshire, UK. ${ }^{3}$ Consultant Anaesthesiologist, University of KwaZulu-Natal, KwaZulu-Natal, South Africa. 
Received: 25 May 2019 Accepted: 16 December 2019 Published online: 18 February 2020

\section{References}

Alphonsus CS, Govender P, Rodseth RN, Biccard BM. The role of cardiac rehabilitation using exercise to decrease natriuretic peptide levels in nonsurgical patients: a systematic review. Perioperative Medicine. 2019; 8:14.

Anguita M, Esteban F, Castillo JC, Mazuelos F, Lopez-Granados A, Arizon JM, Suarez De Lezo J. Usefulness of brain natriuretic peptide levels, as compared with usual clinical control, for the treatment monitoring of patients with heart failure. Med Clin (Barc). 2010;135(10):435-40.

Beck-da-Silva L, de Bold A, Fraser M, Williams K, Haddad H. BNP-guided therapy not better than expert's clinical assessment for beta-blocker titration in patients with heart failure. Congest Heart Fail. 2005;11(5):248-53 quiz 254-245.

Berger R, Moertl D, Peter S, Ahmadi R, Huelsmann M, Yamuti S, Wagner B, Pacher R. N-terminal pro-B-type natriuretic peptide-guided, intensive patient management in addition to multidisciplinary care in chronic heart failure a 3-arm, prospective, randomized pilot study. J Am Coll Cardiol. 2010;55(7):645-53.

Botto F, Alonso-Coello P, Chan MT, Villar JC, Xavier D, Srinathan S, Guyatt G, Cruz P, Graham M, Wang CY, et al. Myocardial injury after noncardiac surgery: a large, international, prospective cohort study establishing diagnostic criteria, characteristics, predictors, and 30-day outcomes. Anesthesiology. 2014;120(3):564-78.

Carubelli V, Lombardi C, Lazzarini V, Bonadei I, Castrini Al, Gorga E, Richards AM, Metra M. $\mathrm{N}$-terminal pro-B-type natriuretic peptide-guided therapy in patients hospitalized for acute heart failure. J Cardiovasc Med (Hagerstown). 2016;17(11):828-39.

Devereaux PJ, Chan MT, Alonso-Coello P, Walsh M, Berwanger O, Villar JC, Wang CY, Garutti Rl, Jacka MJ, Sigamani A, et al. Association between postoperative troponin levels and 30-day mortality among patients undergoing noncardiac surgery. JAMA. 2012;307(21):2295-304.

Duceppe E, Parlow J, MacDonald P, Lyons K, McMullen M, Srinathan S, Graham M, Tandon V, Styles K, Bessissow A, et al. Canadian Cardiovascular Society Guidelines on Perioperative Cardiac Risk Assessment and Management for Patients Who Undergo Noncardiac Surgery. Can J Cardiol. 2017;33(1):17-32.

Eurlings LW, van Pol PE, Kok WE, van Wijk S, Lodewijks-van der Bolt C, Balk AH, Lok DJ, Crijns HJ, van Kraaij DJ, de Jonge N, et al. Management of chronic heart failure guided by individual N-terminal pro-B-type natriuretic peptide targets: results of the PRIMA (Can PRo-brain-natriuretic peptide guided therapy of chronic heart failure IMprove heart fAilure morbidity and mortality?) study. J Am Coll Cardiol. 2010;56(25):2090-100.

Felker GM, Anstrom KJ, Adams KF, Ezekowitz JA, Fiuzat M, Houston-Miller N, Januzzi JL Jr, Mark DB, Pina IL, Passmore G, et al. Effect of Natriuretic PeptideGuided Therapy on Hospitalization or Cardiovascular Mortality in High-Risk Patients With Heart Failure and Reduced Ejection Fraction: A Randomized Clinical Trial. JAMA. 2017;318(8):713-20.

Guyatt GH, Rennie D, Meade MO, Cook DJ. Users' guides to the medical literature: A manual for evidence-based clinical practice. New York: McGrawHill Education; 2015.

Januzzi JL Jr, Rehman SU, Mohammed AA, Bhardwaj A, Barajas L, Barajas J, Kim HN, Baggish AL, Weiner RB, Chen-Tournoux A, et al. Use of amino-terminal pro-Btype natriuretic peptide to guide outpatient therapy of patients with chronic left ventricular systolic dysfunction. J Am Coll Cardiol. 2011;58(18):1881-9.

Jourdain P, Jondeau G, Funck F, Gueffet P, Le Helloco A, Donal E, Aupetit JF, Aumont MC, Galinier M, Eicher JC, et al. Plasma brain natriuretic peptideguided therapy to improve outcome in heart failure: the STARS-BNP Multicenter Study. J Am Coll Cardiol. 2007;49(16):1733-9.

Karlstrom P, Alehagen U, Boman K, Dahlstrom U, group UP-s. Brain natriuretic peptide-guided treatment does not improve morbidity and mortality in extensively treated patients with chronic heart failure: responders to treatment have a significantly better outcome. Eur J Heart Fail. 2011;13(10):1096-103.

Khan MS, Siddiqi TJ, Usman MS, Sreenivasan J, Fugar S, Riaz H, Murad MH, Mookadam F, Figueredo VM. Does natriuretic peptide monitoring improve outcomes in heart failure patients? A systematic review and meta-analysis. Int J Cardiol. 2018;263:80-7.

Lainchbury JG, Troughton RW, Strangman KM, Frampton CM, Pilbrow A, Yandle TG, Hamid AK, Nicholls MG, Richards AM. N-terminal pro-B-type natriuretic peptide-guided treatment for chronic heart failure: results from the BATTLESCARRED (NT-proBNP-Assisted Treatment To Lessen Serial Cardiac Readmissions and Death) trial. J Am Coll Cardiol. 2009;55(1):53-60.

Maeder MT, Rickenbacher P, Rickli H, Abbuhl H, Gutmann M, Erne P, Vuilliomenet A, Peter M, Pfisterer M, Brunner-La Rocca HP, et al. N-terminal pro brain natriuretic peptide-guided management in patients with heart failure and preserved ejection fraction: findings from the Trial of Intensified versus standard medical therapy in elderly patients with congestive heart failure (TIME-CHF). Eur J Heart Fail. 2013;15(10):1148-56.

Moher D, Liberati A, Tetzlaff J, Altman DG, Group P. Preferred reporting items for systematic reviews and meta-analyses: the PRISMA statement. BMJ. 2009;339: b2535.

Murdoch DR, McDonagh TA, Byrne J, Blue L, Farmer R, Morton JJ, Dargie HJ. Titration of vasodilator therapy in chronic heart failure according to plasma brain natriuretic peptide concentration: randomized comparison of the hemodynamic and neuroendocrine effects of tailored versus empirical therapy. Am Heart J. 1999;138(6 Pt 1):1126-32.

Ozkara A, Turgut F, Selcoki Y, Karanfil A, Metin MR, Kanbay M, Tekin O, Akcay A. Probrain natriuretic peptide for assessment of efficacy in heart failure treatment. Adv Ther. 2007;24(6):1233-9.

Persson H, Erntell H, Eriksson B, Johansson G, Swedberg K, Dahlstrom U. Improved pharmacological therapy of chronic heart failure in primary care: a randomized Study of NT-proBNP Guided Management of Heart Failure-SIGNAL-HF (Swedish Intervention study--Guidelines and NT-proBNP AnaLysis in Heart Failure). Eur J Heart Fail. 2010;12(12):1300-8.

Pfisterer M, Buser P, Rickli H, Gutmann M, Erne P, Rickenbacher P, Vuillomenet A, Jeker U, Dubach P, Beer H, et al. BNP-guided vs symptom-guided heart failure therapy: the Trial of Intensified vs Standard Medical Therapy in Elderly Patients With Congestive Heart Failure (TIME-CHF) randomized trial. JAMA. 2009;301(4):383-92.

Puelacher C, Lurati Buse G, Seeberger D, Sazgary L, Marbot S, Lampart A, Espinola J, Kindler C, Hammerer A, Seeberger E, et al. Perioperative Myocardial Injury After Noncardiac Surgery: Incidence, Mortality, and Characterization. Circulation. 2018;137(12):1221-32.

Pufulete M, Maishman R, Dabner L, Mohiuddin S, Hollingworth W, Rogers CA, Higgins J, Dayer M, Macleod J, Purdy S, et al. Effectiveness and costeffectiveness of serum B-type natriuretic peptide testing and monitoring in patients with heart failure in primary and secondary care: an evidence synthesis, cohort study and cost-effectiveness model. Health Technol Asses. 2017;21(40):1.

Rodseth RN, Biccard BM, Le Manach Y, Sessler DI, Lurati Buse GA, Thabane L, Schutt RC, Bolliger D, Cagini L, Cardinale D, et al. The prognostic value of pre-operative and post-operative B-type natriuretic peptides in patients undergoing noncardiac surgery: B-type natriuretic peptide and $\mathrm{N}$-terminal fragment of pro-B-type natriuretic peptide: a systematic review and individual patient data meta-analysis. J Am Coll Cardiol. 2014;63(2):170-80.

Schou M, Gustafsson F, Videbaek L, Andersen H, Toft J, Nyvad O, Ryde H, Fog L, Jensen JC, Nielsen $\mathrm{OW}$, et al. Adding serial $\mathrm{N}$-terminal pro brain natriuretic peptide measurements to optimal clinical management in outpatients with systolic heart failure: a multicentre randomized clinical trial (NorthStar monitoring study). Eur J Heart Fail. 2013;15(7):818-27.

Shah MR, Califf RM, Nohria A, Bhapkar M, Bowers M, Mancini DM, Fiuzat M, Stevenson LW, O'Connor CM. The STARBRITE trial: a randomized, pilot study of B-type natriuretic peptide-guided therapy in patients with advanced heart failure. J Card Fail. 2011;17(8):613-21.

Stienen S, Salah K, Moons AH, Bakx AL, van Pol P, Kortz RAM, Ferreira JP, Marques I, Schroeder-Tanka JM, Keijer JT, et al. NT-proBNP (N-Terminal pro-B-Type Natriuretic Peptide)-Guided Therapy in Acute Decompensated Heart Failure: PRIMA II Randomized Controlled Trial (Can NT-ProBNPGuided Therapy During Hospital Admission for Acute Decompensated Heart Failure Reduce Mortality and Readmissions?). Circulation. 2018; 137(16):1671-83.

Troughton RW, Frampton CM, Yandle TG, Espiner EA, Nicholls MG, Richards AM. Treatment of heart failure guided by plasma aminoterminal brain natriuretic peptide (N-BNP) concentrations. Lancet. 2000;355(9210):1126-30.

Wan X, Wang W, Liu J, Tong T. Estimating the sample mean and standard deviation from the sample size, median, range and/or interquartile range. BMC Med Res Methodol. 2014;14:135.

Weiser TG, Regenbogen SE, Thompson KD, Haynes AB, Lipsitz SR, Berry WR, Gawande AA. An estimation of the global volume of surgery: a modelling strategy based on available data. Lancet. 2008;372(9633):139-44.

\section{Publisher's Note}

Springer Nature remains neutral with regard to jurisdictional claims in published maps and institutional affiliations. 